DOI: 10.31695/IJASRE.2022.8.1.6

\title{
A Comparative Study of Assistive Technologies for Physically challenged peoples for usability of Powered Wheelchair Mobility Aid
}

\author{
Florian Mwijage Rwegoshora ${ }^{1}$ Judith Leo $^{2}$ and Shubi Kaijage \\ Scholar $^{1}$, Lecturer $^{2}$, Senior lecturer ${ }^{3}$ \\ School of Computational and Communication Sciences and Engineering \\ Nelson Mandela African Institution of Science and Technology
}

Arusha-Tanzania

\begin{abstract}
Physical disabilities have always been a big issue in our communities. Ageing, sickness, and other variables have all had a role in the creation of these issues. That is why Powered wheelchairs were designed to aid people with physical disabilities. Wheelchair users have been exposed to a variety of assistive technologies designed to improve their mobility. As a result, different assistive technologies have recently played a significant role in assisting wheelchair users with movement, this is because technology changes so quickly. The recent trendy of assistive technologies include the joystick, brain-computer interface, voice recognition, tongue drive system, eye tracer, and sip and puff. However, some of the most beneficial assistive technologies become difficult to utilize due to technological gaps among individuals in particular nations. The objective of this research to study and review the comparative study of these assistive technologies for Physical Disabilities. In the study, tongue drive system, eye tracer, voice recognition, and sip and puff technologies are compared to joystick assistive technology. The comparison is made based on selected parameters including usability commands, fatigue, response time, information transfer rate, effects, and costs. Based on review, the researchers propose the design of appropriate wheelchairs with assistive technology for developing countries.
\end{abstract}

Key Words: Physical Disabilities, Powered Wheelchair, Assistive Technologies, Developing Countries.

\section{INTRODUCTION}

Individuals with significant physical disabilities, such as those suffering from tetraplegia, a state in which all four limbs are paralyzed, rely largely on assistive technologies for the mobility of wheelchairs in order to improve their quality of life and live more actively and freely [1]. Wheelchairs are becoming more useful and appealing requirements as the life expectancy of the elderly and disabled increases around the world. It is a convenient and desired device by these individuals; as it promotes their freedom and the quality of their lives [2].

Previous studies compared assistive devices in order to aid wheelchair users in having flexible wheelchairs that can provide movement even in difficult areas by altering the mechanical elements of the wheelchairs [3]. This is because individuals with physical disabilities can benefit from assistive technologies to live a self-sufficient, autonomous existence. Without, these assistive tools, people with severe physical disabilities find it difficult to go around daily without constant support. Therefore, in order to effectively enhance the suitability of assistive technologies and users' mobility, users' preferred level of satisfaction, goals and required level of independence should be communicate or collected [4]. In addition, features or term such as usability commands, fatigues, information transmission rate, response time, effects, and costs, we intended to make a good comparison between the joystick and other assistive technologies.

According to the literature, it has been observed that the number of people who are crippled as a result of accidents is growing every day and most of existing assistive technologies or mechanisms are not effective enough to assist users 
especially in developing countries context [5]. Therefore, this paper aims at explaining the essential parameters for assistive tools particularly the wheelchair. These parameters include usability commands, response time, fatigue, information transmission rate, effects, and costs. Then, conduct comparative study between assistive technologies and joystick assistive technology. Lastly, explain the results obtained from the comparative study and then, shows the resulted proposed design of required powered wheelchair for people with disabilities. The following is how the rest of the paper is organized; following the introduction, the next section explains about related studies on assistive technologies, followed by comparison on various parameters of assistive technology, then section for results and discussion and lastly, conclusion part.

\section{RELATED STUDIES ON ASSISTIVE TECHNOLOGIES}

\subsection{Tongue drive system}

Tongue Drive System (TDS) is an assistive technology that uses a wireless network to help persons with a variety of physical limitations to drive wheelchairs and manage different settings [6]. Patients who have lost function of their arms and legs, known as tetraplegia or quadriplegia, may benefit from the Tongue Drive System. The system is described as follows. A magnetic tracer is attached to the tongue, which generates a magnetic field, then there's a headset with an array of three-axis magnetic sensors to monitor changes in the tracer's magnetic field [6]. The magnetic tracer can be mounted to the tongue in two methods. The first is with tissue adhesives like PeriAcryl, which is routinely used in dentistry. However, because tracer tends to loosen after 1 to 2 hours, tongue piercing is an alternative approach [7].

The changes are registered by sensors in the headset, which are then sent to a smartphone or computer, where they are converted into commands for the wheelchair motors. The wheelchair will shift direction as the patient moves his tongue. The tongue drive system has six commands, four of which are directional (UP, DOWN, LEFT, RIGHT) and two of which are selecting commands (single click and double click) [6]. However, it is difficult for persons with limited technological skills to use this system; for example, the process of selecting commands necessitates users to have sufficient technological expertise, which is still a challenge especially in developing countries.

\subsection{Eye tracer}

Measuring using the gaze points is a technique for converting the required movement [8]. The user will need to calibrate his eye location using a predetermined calibration program when using an eye tracker that uses infrared technology to track user gaze location. Patients who have trouble using their limbs are restricted in their mobility. The equipment that can be used for eye tracking is called eye tribe [9]. Electrooculography and image processing are the two most common methods for determining eye gaze. The corneo-retinal standing potential, which exists between the front and back of the human eye, is measured via electrooculography.

The ideal method for determining the user's eye gaze in a wheelchair is to employ a non-contact method based on image processing. Only one individual will be used to determine the gazing points, but if a second user attempts to use the wheelchair, it will be rejected. Simple commands such as forward, backward, left rotation, right rotation, and stop movement are available to the user. On the screen, command labels are presented. The length of time spent at the gazing point is determined by the user's needs [10].

Also, the same technological gap exists in this system because for users to be able to pick instructions, they must be knowledgeable about technology, which is an issue in developing nations with low literacy rates.

\subsection{Sip and puff}

This technique is used by the majority of people who are unable to use their hands. Breath control is the most important approach in this type of technology, and commands are made by sipping and puffing [11]. This design makes use of a Sip-and-Puff device as user input. Sip-and-Puff devices use air pressure from the person's mouth to generate signals that hardware and software can understand [12].A digital signal is generated by a series of sips and puffs, which is processed by a controller to safely propel the wheelchair down the chosen path [13].

The peripipe has an atmospheric pressure sensor that detects changes in air pressure. The pipe detects when the user takes a puff, double-puff, sip, double-sip, or long puff or long sip action based on these changes and wirelessly deliver 
instructions to facilitate wheelchair mobility [11]. The peripipe is a pipe-shaped device that allows users to engage by controlling their breathing.

The technological gap is not very large, but the user must memorize the sip and puffs in order to issue commands, which can be written down or visualized on a computer screen.

\subsection{Brain-computer interface}

The brain computer interface (BCI) is made up of brain neurons, which are important players in this system. The procedure of electroencephalography is used to record the electrical activities of the brain (EEG). The brain signals are controlled in BCI with EEG. The technique is particularly useful for individuals with Amyotrophic Lateral Sclerosis who need to manoeuvre their wheelchair in any direction [14]. The brain signal must be processed and converted into specific commands before the output device can perform the needed tasks. With the assistance of a neurosky sensor that calculates the electrical activity of brain neurons. The electroencephalography (EEG) system is used by the sensor. The billions of cells in your cerebrum produce tiny electrical signals called brainwaves that structure nonstraight instances [15].

Dealing with brain neurons is a massive undertaking that necessitates the user's understanding of the system, which is still a difficulty for individuals due to technology.

\subsection{Voice recognition}

Speech is used to control the wheelchair. The voice is acquired by the system using the voice captured module and then compared with the voice recognition module using the predetermined voices stored in the system. One form of the voice recognition system is speaker-dependent, while the other is speaker-independent; one is dependent on a human, while the other requires only the training of words [16]. The wheelchair's microphone is placed on it so that the wheelchair user can provide commands.

Forward movement, left and right turns, and a stop are among the basic movement functions. The speech recognition processor must be trained with the word uttered out by the user who will operate the wheelchair in order to recognize spoken words [17].

The technology isn't a problem because the words are chosen based on the user's preference for their original tongue.

\subsection{Joystick}

Using the joystick is when the command is started to be initiated and then send to the microcontroller to execute the command. After the execution, the command is sent by the controller in the form of a digital signal to the motor driver to control the movement of the two dc motors. Then due to the command of the joystick, the two-dc motor will rotate [18].

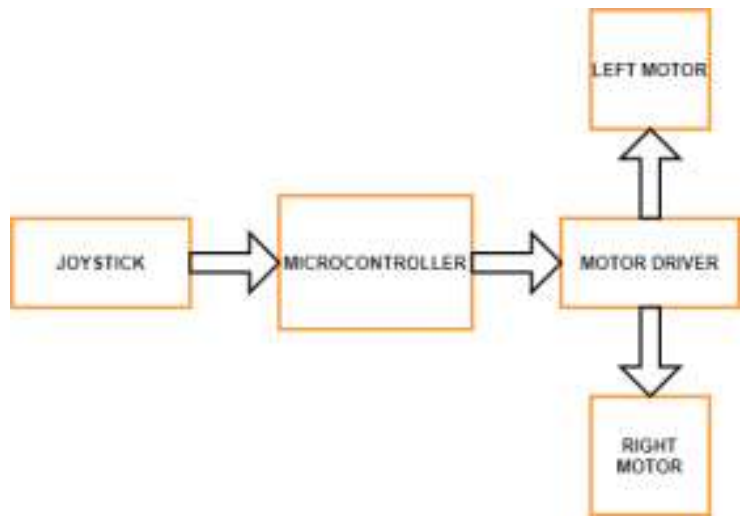

Figure 3.6: Basic block diagram of joystick control for powered wheelchair [18] 
The technology gap isn't a problem because utilizing the joystick is a simple process. The method of directing the wheelchair user is user-friendly.

We can now compare the various features of these assistive technologies to the joystick assistive technology after seeing all of them. So that we can observe the reasons that have led to the rise in popularity of joystick assistive technology today.

\section{COMPARISON OF VARIOUS PARAMETERS FOR ASSISTIVE TECHNOLOGIES}

Based on the literature review, the following parameters were taken into account to obtain different distinctions for assistive technologies.

\subsection{Usability commands}

Because the commands to drive the wheelchair are supplied via the tongue, when the person wishes to undertake regular tongue activities in the middle of the mobility process, this interference tends to cause abnormalities. Particularly when the tongue is in the resting position, which is linked to the neutral instruction [7].

Also, because movement in the eye tracer is reliant on eye command, if the user does not concentrate on the controlling zone, the wheelchair will not move [8].

In terms of supplying commands for easy navigation, Sip and Puff has been recognized as straightforward and easy [19]. This is based on the reality that only the user's breathing status can impact their ability to give commands, while the rest of the system is simple to use.

When using voice recognition, it can be difficult to tell the difference between a typical conversation and a command, which can cause movement issues [20]. Errors in system commands can be caused by daily user conversations.

Because the human body contains billions of neurons that are firing diverse signals at any same moment, obtaining precise signals for wheelchair commands remains a difficulty

However, in the instance of Joystick, it is incredibly simple to navigate and gives commands in a user-friendly manner and does not need the use of a complicated command mechanism [21].

\subsection{Fatigue}

The advantage of TDS is that it eliminates muscle fatigue [22]. Due to the muscles that form the tongue thus it is flexible to operate without feeling any tiredness.

Long-term use of the eye tracer may affect the user's vision due to fatigue from the required attention on the gaze points [23]. The presence of infrared lights causes the user to spend some time selecting commands, which is the main source of fatigue.

After lengthy use, the sip and puff are cumbersome to use, producing fatigue and discomfort [12]. The main cause is that the individual's sips and puffs interfere with his or her natural breathing.

One of the current challenges in $\mathrm{BCI}$ is that controlling a wheelchair for an extended time can put a lot of mental strain on the user, especially for disabled people [24].

Fatigue is not an issue in a speech recognition system because the words are kept short and the quantity is kept to a minimum to keep the system as simple as practical [17].

The other reason that joystick is chosen is since the system has less fatigue compared with other systems [25]. This is all based on the design operation of the joystick that is very straightforward.

\subsection{Response time}

The assessment of these technologies' response time is based on the average response time that can occur within any type of command, as shown in the table below. It's crucial to know how the systems are responding once commands have been executed. According to [26], the BCI's response time appears to be slightly delayed almost 6 to 8 second based on that particular finding.

But the TDS tend to have a quite fast response time of 1 to 3.5 seconds [26]. 
The author also compares the time it takes for the eye tracer and voice system to respond to different commands in this research of which the 2.5 to 3.4 bit second and 2.8 to 3.5 bit second response time were obtained respectively which indicates a moderate response time [27].

Even while sip and puff is inexpensive, it is extremely slow to respond [28].

For expert users, the joystick has a response time of roughly 0.3 to 1 second while covering various movements. The response time when using a joystick is quite quick, and it is possible to move in 360 degrees in a short time [29].

\subsection{Information transfer rate}

The information transmission rate (ITR) measures how much information is contained in a communication channel's output signal in comparison to the input signal. The human-interface system can be thought of as a communication channel in these investigations, with the human having to send a movement intention through the interface [30].

The table's results were based on a certain number of participants, and the transmission rate for BCI and TDS was dete rmined to be a little low as indicated [26]. The ITR was determined with the participation of at least 16 people, and in the case of the eye tracer, the information transfer rate varies depending on whether the track is simple or complex [9].

The situation with voice recognition The rate at which information is transferred varies depending on the circumstances, but it is estimated to be roughly 1.8 bits per second for simple commands [31].

However, based on these data, joysticks tend to have a good ITR of roughly 2.1 bits per second [29].

\subsection{Effects}

Table 1 shows that because BCIs involve brain neurons, there is a high risk of users developing a cerebral tumour [14].

The usage of an eye tracer can also cause eye damage due to infrared [14].

To avoid disease transmission, the Sip and Puff method requires that the tubes be kept clean at all times and due to the presence of magnetic tracer, Electromagnetic interference must be suppressed [7].

Because voice recognition systems rely on the use of voice, they can cause interference in noisy environments [14].

However, the presence of a magnetic tracer in TDS is uncomfortable for users [14].

When it comes to joysticks, the most crucial factor to consider is the location where the joystick will be put. As a result, it's often mounted on the armrest to avoid unnecessary effects.

\subsection{Cost}

When it comes to cost, TDS and BCI are two of the most expensive assistive technologies available. Consumers with limited financial resources will find it difficult to obtain this technology [14].

One of the most important aspects to think about is affordability. It has limitations because of the diaphragm control, but the sip and puff technology is inexpensive and easy to use [7].

Voice recognition and eye-tracking devices have also been discovered to be low-cost assistive technology [14].

However, aside from being a low-cost technology, joystick assistive technology is the most often used [3].

\section{RESULTS AND DISCUSSION}

\subsection{Results}

Table 4.1 Comparison of the assistive technologies with respect to the parameters

\begin{tabular}{lllll}
\hline Type & $\begin{array}{l}\text { Average } \\
\text { Response } \\
(\mathbf{s})\end{array}$ & $\begin{array}{l}\text { Information } \\
\text { Transmission } \\
\text { rate }(\mathbf{b i t} / \mathbf{s e c})\end{array}$ & Cost & Effects \\
\hline EEG-BCI & $6.0-8.0$ & 0.42 & Expensive & Can cause cerebral tumor \\
\hline TDC & $1-3.5$ & 1.26 & Expensive & Not comfortable \\
\hline $\begin{array}{l}\text { Voice } \\
\text { recognition }\end{array}$ & $2.8-3.5$ & $1.8-3.6$ & $\begin{array}{l}\text { Not } \\
\text { expensive }\end{array}$ & $\begin{array}{l}\text { Errors in recognition can be } \\
\text { caused by noise. }\end{array}$ \\
\hline system & & & Not & Infrared LEDs cause damage to \\
\hline
\end{tabular}


International Journal of Advances in Scientific Research and Engineering (ijasre), Vol 8 (1), January -2022

\begin{tabular}{lccll}
\hline Sip and Puff & $5.0-8.0$ & 0.5 & expensive & the eyes. \\
\hline Joystick & $0.3-1$ & 2.1 & $\begin{array}{l}\text { Not } \\
\text { Expensive }\end{array}$ & $\begin{array}{l}\text { Tubes must be kept clean at all } \\
\text { times. }\end{array}$ \\
\hline
\end{tabular}

\subsection{Discussion}

Based on the findings, the study recommends that the following elements be included in the design of an acceptable powered wheelchair with assistive technology for developing countries:

- For the wheelchair's and the wheelchair user's safety, a location monitoring feature must be included.

- To aid in the fire escape, a fire detection feature must be provided.

- Wheelchair users must be able to send SMS notifications to their loved ones.

- A wheelchair must be capable of avoiding nearby obstructions.

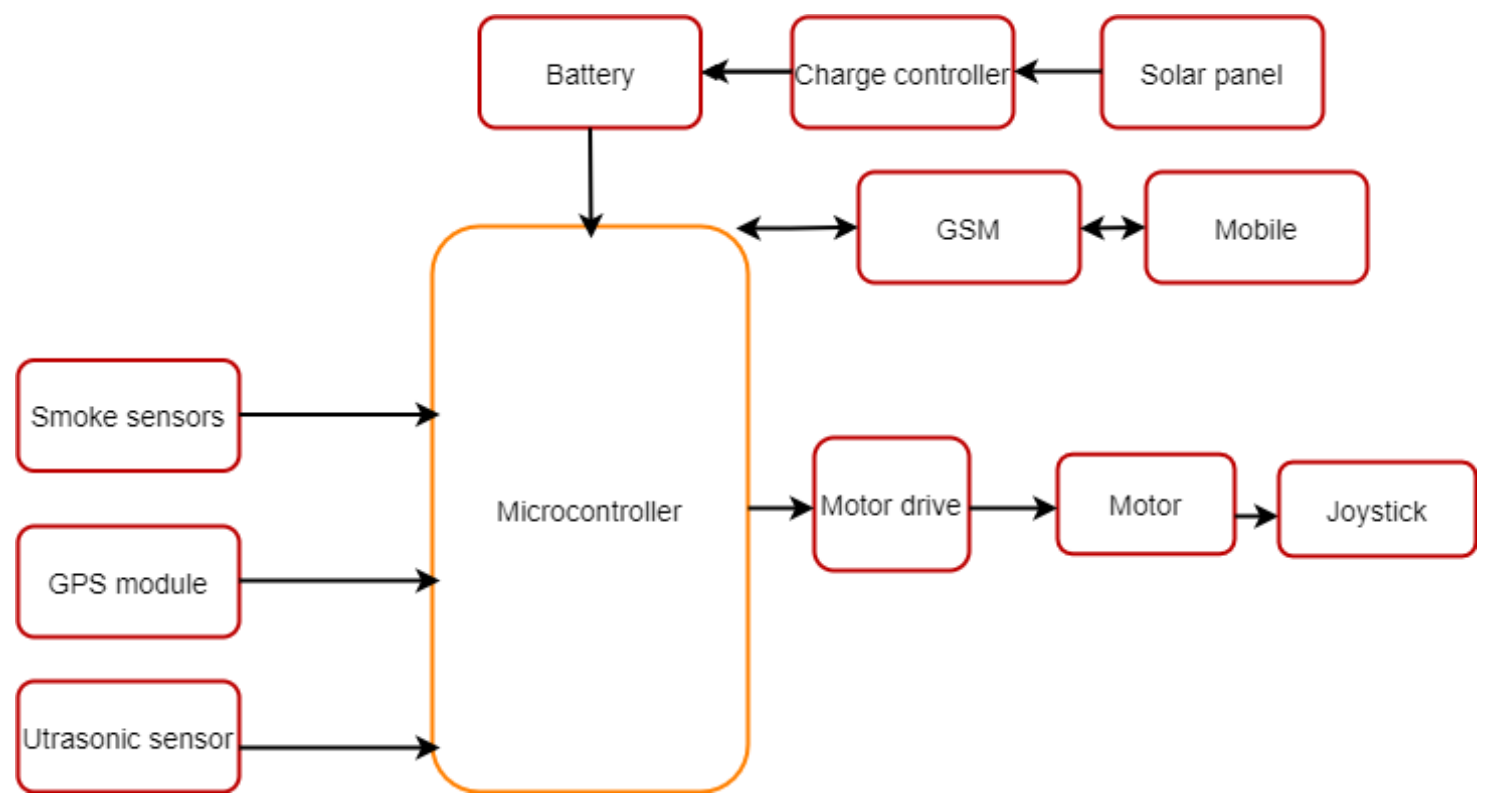

Figure 4.2: Proposed system block diagram

The proposed wheelchair will provide the following advantages to users, particularly those in developing countries.

- With the wheelchair user's permission, the wheelchair user's position will be easily traced in the event of a problem.

- When a fire occurs, the wheelchair user will be alerted by a buzzer sound, and his close family will be alerted by a message. This raises the chances of the wheelchair user escaping while also lowering the risk.

- In the incidence of a problem, the wheelchair user can simply press the button to request assistance from people whose phone numbers have been synchronized with the system.

- One of the wheelchair's functions will be to make it easier to detect nearby obstacles.

\section{CONCLUSION}

People with physical disabilities rely on assistive technologies to help them move around in their daily lives. The researchers and developers of assistive technologies should ensure that the manufactured wheelchairs fulfill users requirements and are appropriate for a specific society, country or continent. This is due to the fact, most users especially who resides in developing countries cannot purchase expensive wheelchairs such as wheelchairs with joysticks. In this study, we have been able to examined the context in which assistive technologies are used and compared it to joystick assistive technology. This research found there are so different ways on how innovatively assistive technology can be intergrated by properly taking users' requirements, considering the nature of the country or 
environment, and choosing appropriate parameters, developers around the world can be able to design as well develop effective powered wheelchair to aid different users' mobility. In this study, we have proposed suitable design of powered wheelchair which is integrated with assistive technologies. The study expects to develop and validate the proposed design and also, recommends other researchers in other countries to review the different existing types of wheelchairs in order aid effective mobility to wheelchair users.

\section{REFERENCES}

[1] A. Jafari, N. Buswell, M. Ghovanloo, and T. Mohsenin, systems. A low-power wearable stand-alone tongue drive system for people with severe disabilities. 2017;12(1):58-67.

[2] Y. Rabhi, M. Mrabet, F. Fnaiech, and P. Gorce, editors. Intelligent joystick for controlling power wheelchair navigation. 3rd International Conference on Systems and Control; 2013: IEEE.

[3] P. Arefin, MS. Habib, A. Arefin, and M. Arefin. A comparison of mobility assistive devices for elderly and patients with lower limb injury: Narrative Review. 2020;2(1):13-7.

[4] A. Agrawal, AB. Varshney, MK. Rai, and H. Garg, Engineering. Smart Automation Using Tongue Motion. 2020;1(4):24550.

[5] H. Krishna, S. Emani, R. Manapaka, and SS. Rao. VOICE CONTROLLED AND JOYSTICK BASED WHEEL CHAIR FOR DIFFERENTLY ABLED PEOPLE.

[6] B. Yousefi, X. Huo, and M. Ghovanloo, editors. Preliminary assessment of Tongue Drive System in medium term usage for computer access and wheelchair control. 2011 Annual International Conference of the IEEE Engineering in Medicine and Biology Society; 2011: IEEE.

[7] J. Kim, X. Huo, J. Minocha, J. Holbrook, A. Laumann, and M. Ghovanloo. Evaluation of a smartphone platform as a wireless interface between tongue drive system and electric-powered wheelchairs. 2012;59(6):1787-96.

[8] S. Plesnick, D. Repice, and P. Loughnane, editors. Eye-controlled wheelchair. 2014 IEEE Canada International Humanitarian Technology Conference-(IHTC); 2014: IEEE.

[9] YK. Meena, A. Chowdhury, H. Cecotti, K Wong-Lin, SS. Nishad, and A. Dutta, et al., editors. Emohex: An eye tracker based mobility and hand exoskeleton device for assisting disabled people. 2016 IEEE International Conference on Systems, Man, and Cybernetics (SMC); 2016: IEEE.

[10]D. Cojocaru, LF. Manta, IC. Vladu, A. Dragomir, and AM. Mariniuc, editors. Using an eye gaze new combined approach to control a wheelchair movement. 2019 23rd International Conference on System Theory, Control and Computing (ICSTCC); 2019: IEEE.

[11]T. Feldt, S. Freilich, S. Mendonsa, D. Molin, and A. Rau, editors. The peripipe: A sip-and-puff remote control for music playback. Proceedings of the International Conference on New Interfaces for Musical Expression (NIME); 2015.

[12] HS. Grewal, A. Matthews, R. Tea, V. Contractor, and K. George, editors. Sip-and-Puff Autonomous Wheelchair for Individuals with Severe Disabilities. 2018 9th IEEE Annual Ubiquitous Computing, Electronics \& Mobile Communication Conference (UEMCON); 2018: IEEE.

[13] I. Mougharbel, R. El-Hajj, H. Ghamlouch, and E. Monacelli, editors. Comparative study on different adaptation approaches concerning a sip and puff controller for a powered wheelchair. 2013 Science and Information Conference; 2013: IEEE.

[14] A. Ghorbel, NB. Amor, and M. Jallouli . A survey on different human-machine interactions used for controlling an electric wheelchair. 2019;159:398-407.

[15] H. Sharma, R. Mahajan, G. Sakthivel, D. Saravanakumar, and N. Raghukiran, editors. Brain Computer Interface Controlled Wheel Chair. Journal of Physics: Conference Series; 2021: IOP Publishing. 
[16] N. Aktar, I. Jaharr, and B. Lala, editors. Voice recognition based intelligent wheelchair and GPS tracking system. 2019 International Conference on Electrical, Computer and Communication Engineering (ECCE); 2019: IEEE.

[17]C. Priya, S. Pranjala, and H. Supreeth, Technology E. Voice Controlled Wheelchair for Physically Disabled People. 2018;6:2375-80.

[18] T. Saharia, J. Bauri, and C. Bhagabati, Technology. Joystick controlled wheelchair. 2017;4(7).

[19]BE. Dicianno, RA. Cooper, and J. Coltellaro, America rcoN. Joystick control for powered mobility: Current state of technology and future directions. 2010;21(1):79.

[20] S. Rojhani, SA. Stiens, and A. Recio. Independent sailing with high tetraplegia using sip and puff controls: integration into a community sailing center. 2017;40(4):471-80.

[21] S. Suryawanshi, J. Chitode, and S. Pethakar, Engineering S. Voice operated intelligent wheelchair. 2013;3(5):487-90.

[22] JH. Choi, Y. Chung, and S. Oh. Motion control of joystick interfaced electric wheelchair for improvement of safety and riding comfort. 2019;59:104-14.

[23] MN. Sahadat, N. Sebkhi, D. Anderson, and M. Ghovanloo. Optimization of tongue gesture processing algorithm for standalone multimodal tongue drive system. 2018;19(7):2704-12.

[24] MK. Shahin, A. Tharwat, T. Gaber, and A. Hassanien. A wheelchair control system using human-machine interaction: singlemodal and multimodal approaches. 2019;28(1):115-32.

[25] R. Zhang, Y. Li, Y. Yan, H. Zhang, S. Wu, and T. Yu, et al. Control of a wheelchair in an indoor environment based on a brain-computer interface and automated navigation. 2015;24(1):128-39.

[26] X. Huo, J. Wang, and M. Ghovanlo, Development. Introduction and preliminary evaluation of the tongue drive system: Wireless tongue-operated assistive technology for people with little or no upper-limb function. 2008;45(6).

[27] S. Anwer, A. Waris, H. Sultan, SI. Butt, MH. Zafar, and M. Sarwar, et al. Eye and voice-controlled human machine interface system for wheelchairs using image gradient approach. 2020;20(19):5510.

[28]X. Huo, and M. Ghovanloo. Evaluation of a wireless wearable tongue-computer interface by individuals with high-level spinal cord injuries. 2010;7(2):026008.

[29] GU. Sorrento, PS. Archambault, F. Routhier, D. Dessureault, and P. Boissy, rehabilitation. Assessment of joystick control during the performance of powered wheelchair driving tasks. 2011;8(1):1-11.

[30] J. Lobo-Prat, AQ. Keemink, AH. Stienen, AC. Schouten, PH. Veltink, B. Koopman, et al. Evaluation of EMG, force and joystick as control interfaces for active arm supports. 2014;11(1):1-13.

[31] CM. Reed, and N. Durlach. Note on information transfer rates in human communication. 1998;7(5):509-18. 